\title{
Evoked response of the human visual cortex: Spectral sensitivity'
}

Carl R. Cavonius

EYE RESEARCH FOUNDATION, BETHESDA, MARYLAND AND HUMAN SCIENCES RESEARCH, MCLEAN, VIRGINIA

\begin{abstract}
Abstraet
Responses evoked by visual stimuli were recorded from the human scalp over the visual cortex. At low intensities response amplitude is linear with log stimulus energy. Spectral sensitivity of the cortical response resembles that of the electroretinogram elicited by similar stimuli but shows maximum sensitivity at about $560 \mathrm{~m} \mu$, indicating a largely photopic origin.
\end{abstract}

\section{Problem}

Psychophysical measurements of the spectral sensitivity of the human visual system generally yield either the photopic or the scotopic C.I.E. spectral sensitivity function, depending upon the observer's state of adaptation and upon stimulus intensity, area and retinal locus. The photopic function (C, Fig. 1) is measured by restricting the stimulus to the rod-free fovea. It exhibits maximum sensitivity at about $560 \mathrm{~m} \mu$, and is generally thought to represent the spectral sensitivity of the cone mediated systems, which signal color and brightness information at moderate and high levels of light adaptation. The scotopic (rod moderated) spectral sensitivity function describes the dark adapted observer's threshold sensitivity to weak extra-foveal stimuli, and peaks at about $510 \mathrm{~m} \mu$.

The spectral sensitivity of the human electroretinogram (ERG) resembles the psychophysical scotopic function when the ERG is elicited by single flashes (Riggs \& Johnson, 1949) or by slowly flickering stimuli (Armington \& Biersdorf, 1956). Predominantly scotopic ERGs are recorded even when the eye is light adapted and when the stimulus is imaged on the fovea (Boynton \& Riggs, 1959) although these same stimulus conditions yield the photopic luminous efficiency function in comparable psychophysical experiments. The unexpected scotopic dominance of the ERG is now generally attributed to the great numerical predominance of rods (the scotopic receptors) in the human retina, and to the fact that light scattered within the eye has been shown to be effective in stimulating receptors outside the intended stimulus area (Riggs, 1958).

In the visual cortex, on the other hand, the projection of the fovea is greatly magnified compared to the projection of the peripheral retina (Holmes, 1945). We therefore predicted that the spectral sensitivity of the primary cortical response evoked by visual stimulation should more nearly resemble the photopic C.I.E. function. The following study tests this prediction.

\section{Method}

Monochromatic stimuli produced by a Bausch \& Lomb grating monochromator were presented in $60^{\circ}$
Maxwellian view to the S's light adapted right eye. Stimuli were flickered at a rate of 4 per sec. with equal light-dark intervals. Maximum stimulus intensity at each wavelength was approximately equal to a directly viewed $7 \mathrm{ft}-\mathrm{L}$. field. The most stable records were obtained when the flickering light was superimposed on a steady white adapting field of approximately the same brightness. Evoked potentials were detected by bipolar chlorided silver disc electrodes applied with bentonite paste to the scalp on the midline over the inion and vertex. Responses were amplified by a condenser coupled amplifier ( $0.1 \mathrm{sec}$. time constant) and were cumulated in a Mnemotron Computer of Average Transients. The $\mathrm{S}$ controlled the computer so that data was only accumulated when he was properly aligned with the optical system.

\section{Results}

At high stimulus intensities the evoked cortical response resembled those shown by Ciganek (1961), except for a small inion-positive wave which preceded Ciganek's wave I. At low intensities only a single monophasic response remains, which appears to correspond with Ciganek's wave III.

When high stimulus intensities are used peak-to-peak amplitude of the evoked potential does not vary monotonically with stimulus intensity (cf. Armington, 1964). However, at moderate intensities a range of about $2 \mathrm{log}$ units was found over which response amplitude is linearly related to $\log$ stimulus intensity (Fig. 2). The mean correlation of determination (Pearson $\mathrm{r}^{2}$ ) for

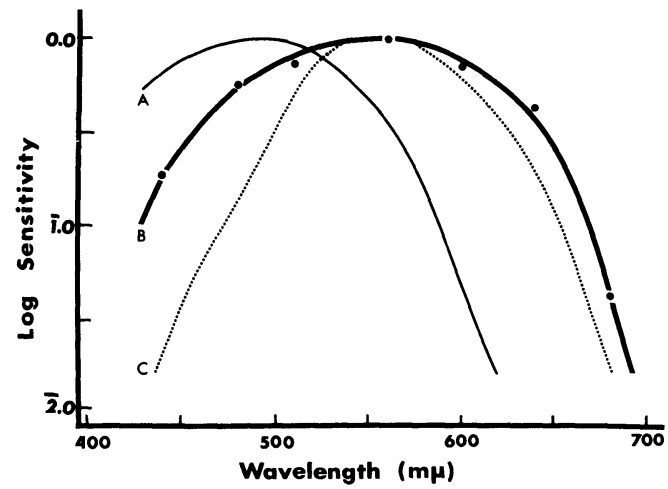

Fig. 1. Spectral sensitivity of the evoked cortical response. A Scotopic ERG sensitivity (Riggs \& Johnson, 1949). B: Cortical response sensitivity. C: C.I.E. photopic relative luminous efficiency function. 


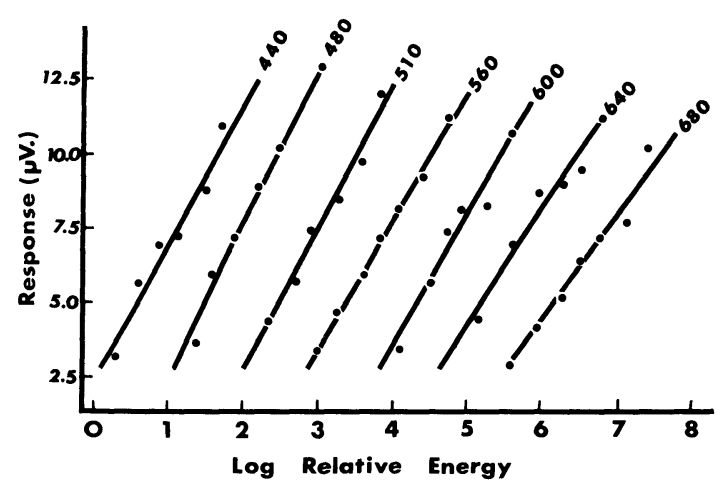

Fig. 2. Amplitude of evoked cortical response vs. stimulus energy. Lines fitted by the method of least squares. For clarity, data at $480 \mathrm{~m} \mu$ is displaced to the right $1.3 \mathrm{log}$ units; data at $510,2.4$ units; $560,3.5$ units; $600,4.3$ units; $640,5.0$ units; $680,6.0$ units. Stimulus wavelength $(\mathrm{m} \mu)$ is given above each line.

this data is 0.96 , which indicates that within this range response amplitude is almost entirely a function of intensity.

The slopes of these functions increase with decreasing wavelength between $640 \mathrm{~m} \mu$ and $480 \mathrm{~m} \mu$. At $440 \mathrm{~m} \mu$ the slope once again decreases. A similar effect has been found in the ERG (Armington \& Biersdorf, 1956).

Sensitivity is conventionally defined by the reciprocal of the relative energy required to elicit a certain response. A $6 \mu \mathrm{V}$ critierion response was used to compute the cortical spectral sensitivy function (B, Fig. 1).It is similar in shape to the ERG sensitivity function (A), but is displaced about $60 \mathrm{~m} \mu$ toward the long wavelength end of the spectrum. This shift of peak sensitivity and the similarity between the right limbs of the cortical sensitivity and the C.I.E. photopic function (C) supports our view that cortical evoked responses elicited by long wavelength stimuli are largely photopic in origin. Below $560 \mathrm{~m} \mu$ sensitivity of the cortical response exceeds the C.I.E. function, which probably indicates that a mixture of photopic and scotopic activity is present in the cortical response. This departure from the C.I.E. function at short wavelengths has also been reported by Armington (1964).

\section{Diseussion}

It is difficult to use the ERG to study the human photopic visual system because of scotopic dominance of the ERG (Cavonius, 1964). The present study suggests an alternative experimental technique, since it shows that photopic responses may be recorded from the region of the visual cortex and that these responses are not just the ERG recorded at a distance. We believe that this response may be used to further explore the nature of the human color vision system, and particularly to see whether evidence for a trichromatic or an opponentcolors mechanism can be detected at the cortical level. In particular, the effect of chromatic adaptation (which has relatively slight effect on the photopic ERG) should be investigated. Recording the cortical response may also make possible routine clinical assessment of the photopic portion of the visual system.

\section{References}

ARMINGTON, J. C. Relations between electroretinogram and occipital potentials elicited by flickering stimuli, Doc. Ophthal., 1964, XVIII, 194-206.

ARMINGTON, J. C., \& BIERSDORF, W. R. Flicker and color adaptation in the human electroretinogram. J. Opt. Soc. Amer., 1956, $46,393-400$.

BOYNTON, R. B., \& RIGGS, L. A. The effect of stimulus area and intensity upon the human retinal response. J. exp. Psychol., 1951, $42,217-266$.

CAVONIUS, C. R. Color sensitive responses in the human flickerERG. Doc. Ophthal., 1964, XVIII, 103-113.

CIGANEK, C. The EEG response (evoked potential) to light stimulus in man. EEG clin. Neurophysiol., 1961, 13, 165-172.

HOLMES, G. The organization of the visual cortex in man. Proc. Roy. Soc. Lond., 1945, B132, 348-361.

RIGGS, L. A. The human electroretinogram. A.M.A. Arch. Ophthal., $1958,60,739-754$.

RIGGS, L. A., \& JOHNSON, E. P. Electrical responses of the human retina. J. exp. Psychol., 1949, 39, 415-424.

Note

1. Ths study was performed at the Department of Psychology, Brown University with the collaboration of Dr. Walter Makous of the Thomas J. Watson Research Center, International Business Machines Corporation. Equipment was provided from funds from the Office of Naval Research, grant number Nonr. 562(21). 